Hamlet und Deutschland 
Franz Loquai

\section{Hamlet und Deutschland}

Zur literarischen Shakespeare-Rezeption im 20. Jahrhundert 
Die Umschlagabbildung zeigt Wolfgang Hildesheimers Collage „Zitatenschatzkammer“ (1969). Der Abdruck erfolgt mit freundlicher Erlaubnis von Silvia Hildesheimer und Volker Jehle.

Die Deutsche Bibliothek - CIP-Einheitsaufnahme

\section{Loquai, Franz:}

Hamlet und Deutschland : zur literarischen Shakespeare-

Rezeption im 20. Jahrhundert / Franz Loquai. - Stuttgart ;

Weimar : Metzler, 1993

(Metzler Studienausgabe)

ISBN 978-3-476-00895-4

ISBN 978-3-476-00895-4

ISBN 978-3-476-03463-2 (eBook)

DOI 10.1007/978-3-476-03463-2

Dieses Werk einschließlich aller seiner Teile ist urheberrechtlich geschützt. Jede Verwertung außerhalb der engen Grenzen des Urheberrechtsgesetzes ist ohne Zustimmung des Verlages unzulässig und strafbar. Das gilt insbesondere für Vervielfältigungen, Übersetzungen, Mikroverfilmungen und die Einspeicherung und Verarbeitung in elektronischen Systemen.

(C) 1993 Springer-Verlag GmbH Deutschland

Ursprünglich erschienen bei J. B. Metzlersche Verlagbuchhandlung und Carl Ernst Poeschel Verlag GmbH in Stuttgart 1993 


\section{INHALT}

1. Vorgeschichte und Themenstellung . . . . . . . . . . . . . 3

a) >Deutschland ist Hamlet< als Gleichung des 19. Jahrhunderts 3

b) Hamlet als Paradigma im 20. Jahrhundert 11

2. Erkenntnisinteresse und Zielsetzung. . . . . . . . . . . . . . 14

a) Zum Begriff des Hamletsyndroms 14

b) Die heuristische Funktion der Modellbildung 15

3. Forschungslage und Textauswahl . . . . . . . . . . . . 18

4. Methodisches Vorgehen . . . . . . . . . . . . . 21

a) Sozialgeschichte versus Bearbeitungstypologien 21

b) Intertextualität und unendlicher Text 24

c) Zur Praxis der Interpretation 29

ERSTER TEIL: HAMLET UND DIE DEUTSCHE MISERE

1. Schuld und Sühne - Hamlet und Raskolnikow.

Ernst Weiß: »Georg Letham, Arzt und Mörder« . . . . . . . . . . . . 34

a) Die letzten Europäer 36

b) Die Torpedierung des Vaters 38

c) Schuld und Sühne 41

d) Das Verschwinden in der Menge 44

2. Die allmähliche Abtötung der Vitalität beim Leben.

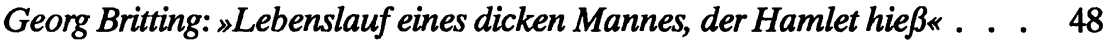

a) Die Wasserleiche 49

b) Hamster, Schwein und Riesenfrosch 51

c) Das große Fressen 54

d) Streunende Katzen 60

e) Hinter der weißen Mauer 62

f) Mutterschoß und Kummerspeck 68 
3. Shakespeares »Hamlet« als Mausefalle für Hauptmann.

Gerhart Hauptmann: „Im Wirbel der Berufungк. . . . . . . . . . . . 72

a) Frühschoppen, Teekränzchen, Kommersabend 75

b) Gotter, Ophelia und Jesus 77

c) Hauptmann ist nicht Hamnet 81

Zusammenfassung: Hamlet als psychologisches Modell

1. Die Germanisierung des nordischen Stoffes.

Gisela Wenz-Hartmann: „Amleth" . . . . . . . . . . . . . . . 90

a) Die Sprache des Nationalsozialismus 95

b) Die Ambivalenz widersprüchlicher Kodierung 96

c) Amleth ist Deutschland 98

2. Hamlet und der Widerstand gegen den Nationalsozialismus.

Klaus Mann: „Mephisto« . . . . . . . . . . . . . . . . . . . . 102

a) Mephisto macht alles 103

b) Der Hofnarr des Dritten Reiches 104

c) Der rächende Geist des Widerstandes 109

Zusammenfassung: Ideologisch-weltanschauliche Modellbildung . . . . 112

1. Auf der Suche nach der Schuld am Krieg. Alfred Döblin: „Hamlet oder Die lange Nacht nimmt ein Ende» . . . . . 115

a) Der trauernde Hamlet 118

b) Entlarvendes Erzählen 120

c) Weltkrieg und Familienkatastrophe 124

d) Schuld und Buße oder die Flucht in die Religion 126

2. Yorick in der $>$ Stunde Null<. Günter Grass: „Die Blechtrommel« . . . . . . . . . . . . . . . . . 129

a) Yoricks Liebe zu den Friedhöfen 130

b) Rosmarin und die Deutschen 132 
3. Hamlet in den Trümmern.

Günter Kunert: „Im Namen der Hüte» . . . . . . . . . . . . . . . 135

a) Die Mütze als Mausefalle 136

b) Das Ende der poetischen Gerechtigkeit 137

4. Hamlet und das Jahr 1933.

Walter Jens: »Herr Meister» ． . . . . . . . . . . . . . . . . . . 141

a) Die Suche nach dem Stoff 141

b) Hamlet und die Trauer in Deutschland 143

c) Der Neuanfang als künstlerische Aufgabe 145

Zusammenfassung: Sozialgeschichtliche Modellbildung . . . . . . . 148

1. Die Unfähigkeit zur Revolte.

Konrad Wünsche: „Der Unbelehrbare« . . . . . . . . . . . . . . . 151

a) Die verweigerte Rache 153

b) Die Ermordung Hamlets 155

2. Die Absage an die Welt der Väter. Martin Walser: „Der schwarze Schwan« . . . . . . . . . . . . 158

a) Hamlets Vater als KZ-Mörder 159

b) Der Selbstmord des Sohnes als Revolte 164

3. Die Abrechnung mit den Vätern . . . . . . . . . . . . . . . . . 167

a) Martin Walser: Hamlet als Autor 167

b) Die Vaterbücher: Der Autor als Hamlet 170

Zusammenfassung: Hamlet als heuristisches Modell . . . . . . . . . . . 176

1. Endspiel im Herzen der Finsternis.

Heiner Müller: „Die Hamletmaschine»

a) Die Liquidierung Shakespeares 182

b) Die Destruktion Hamlets und Ophelias 183

c) Die Exekution des Autors 189 
2. Flucht aus der Geschichte in die Geheimnisse der Kunst.

Wolfgang Hildesheimer und Hamlet. . . . . . . . . . . . . . 192

a) Auf der Suche nach der Wahrheit 194

b) Die Erfindung der Wahrheit 196

c) Ein guter Fund: Hamlet 197

d) Hamlet und die Häscher oder die Fuge vom Tod 200

e) Die Flucht aus der Geschichte und die Geheimnisse in der Seelenmusik des Künstlers 204

f) Schuld und Schweigen 211

Zusammenfassung: Modelle der Distanzierung . . . . . . . . . . 214

NACHSPIEL: DAS WEITERLEBEN HAMLETS

1. Spielen als Lebenskunst . . . . . . . . . . . . . . . . . . . 217

2. Die Kunst als Spiel . . . . . . . . . . . . . . . . . . . . 220

Literaturverzeichnis . . . . . . . . . . . . . . . . . 227

Register.................... . 251 
The Readiness is all

Call me what instrument you will, though you fret me, you cannot play upon me

(Hamlet)

für Mufti 


\section{VORBEMERKUNG}

Die vorliegende Studie ist die geringfügig überarbeitete Fassung einer Untersuchung, die 1991 von der Fakultät Sprach- und Literaturwissenschaften der Otto-Friedrich-Universität Bamberg als Habilitationsschrift angenommen wurde. Mein Dank gilt der Deutschen Forschungsgemeinschaft für das gewährte Stipendium. Für Anregungen, Hinweise und Hilfe möchte ich sehr herzlich danken Wulf Segebrecht sowie Friedhelm Auhuber, Peter Hanenberg, Volker Jehle, Fridrun Rinner, Wolfgang von Ungern-Sternberg und, last but most, meiner Frau. Für die Erstellung des Registers danke ich dem guten Geist Archivarius Aggwyler. 